\title{
Mucosal metaplasia and chronic inflammation in the middle ear of infants receiving intensive care in the neonatal period
}

\author{
D J DESA \\ Department of Pathology, McMaster University Medical Centre, Hamilton, Ontario, Canada
}

SUMMARY The histological findings in the middle ear cavity of 72 infants of varying gestations, birthweights, and ages are presented. All infants died after receiving ventilatory support and oxygen for longer than 14 days. In 5 infants there was no detectable histological abnormality. In the remainder, a wide range of lesions was seen including glandular metaplasia, retained squamous debris, squamous polyps, otitis media, and destruction of ossicles. None of the cases of otitis media was diagnosed before necropsy; all were associated with pneumonia. No single specific infectious agent predominated. Several factors could contribute to the spectrum of lesions, and these include persistent amniotic squamous debris, infection, and the effects of oxygen and a nasal airway. The possible implications of these findings are discussed, and it is argued that similar changes of lesser severity could be present in survivors in whom otitis media and conduction hearing defects could be expected.

Earlier reports have demonstrated that many histological abnormalities may be identified in the middle ear cavities of stillbirths and newborn infants at necropsy. These include persistence of amniotic squamous debris, polyps becoming organised from such debris, metaplastic epithelium, and frank otitis media. ${ }^{1-3}$ In neonatal infants with otitis media no single infectious agent predominates, and, while some of the cases are associated with chorioamnionitis, ${ }^{2}$ this is not an invariable feature. ${ }^{13}$

This report presents the findings, at necropsy, in the middle ear of 72 infants who received ventilatory support and oxygen for at least 14 days in a neonatal intensive care unit. A wide range of metaplastic epithelial changes was present, but in addition the ossicles were damaged in 8 infants.

\section{Materials and methods}

Between 1 January 1973 and 31 December 1981, 72 infants who received prolonged ventilatory support and oxygen for a minimum of 14 days in our neonatal intensive care unit died, and were studied personally at necropsy.

The infants were of varying birthweights, gestations, and ages (Table 1). Most of the infants died while still on the unit. Twelve infants were discharged,
Table 1 Birthweights, gestational and postnatal ages of the 72 infants

\begin{tabular}{lc}
\hline Gestation at delivery (weeks) & $22-42$ (median 27) \\
Birthweight (g) & $580-3650$ (median 980) \\
Postnatal age (days) & $14-296$ (median 38) \\
\hline
\end{tabular}

either to another hospital or to their homes and were readmitted later. Details of the clinical history and progress were available from the infants' hospital records and, with the exception of removing the eyes, a full necropsy was performed in all instances.

The procedure for examination of the petrous temporal bones has been described previously. ${ }^{1}$ The left petrous temporal bone was opened and its contents sampled for microbiological investigation and cytological smears. The right petrous temporal bone was dissected en bloc, fixed in $10 \%$ formalsaline, and decalcified in formic/citric decalcifying fluid. In 71 of the 72 infants, after decalcification, blocks were sampled in the horizontal plane through the external auditory canal and tympanic membrane. ${ }^{1}$ Paraffin-embedded sections were stained primarily with Attwood's stain ${ }^{4}$ and other stains as thought appropriate. The sole exception was the oldest infant in the group. Here the tegmen tympani of the right petrous temporal bone was removed, and the middle 
ear cavity and contents examined from above before decalcification.

\section{Results}

All the infants had pathological lesions in major organ systems, sufficient to explain the cause of death and morbidity. These included bronchopulmonary dysplasia and myocardial scarring, as well as intraventricular haemorrhage and haemorrhagic necrosis of many abdominal viscera. The changes in the middle ear were incidental findings, but since they were neither suspected nor diagnosed clinically, they were of particular interest. In 10 infants the ears had been examined during life, and the tympanic membrane was stated to be intact.

Microbiological findings. All the infants in this series had been treated with antibiotics at some stage, and 8 were receiving antibiotic therapy at the time of their death. However, in 16 infants significant isolates of infectious agents were obtained at necropsy. No single organism predominated. Gramnegative organisms of various types were isolated from 9 infants; Candida albicans was isolated from 2 infants and Haemophilus influenzae, Haemophilus pertussis, group B $\beta$-haemolytic streptococci, Staphylococcus aureus, and cytomegalovirus were isolated from a single infant in each instance.

Histological findings. The findings are summarised in Table 2.

Five infants had no histological abnormality of the middle ear cavities of either left or right petrous temporal bone. All these infants were under 28 days of age and had been born at 26 to 38 weeks' gestation. They acted as controls for the 67 infants who had detectable lesions.

As can be seen (Table 2), metaplastic epithelial changes were present in all 67 infants. The normal medial wall of the middle ear is clothed by mucoperiosteum with a fine, delicate stroma, that is covered by a flattened layer of simple squamous epithelium. In the majority of the infants in this

Table 2 Summary of histological findings

\begin{tabular}{lc}
\hline Abnormality & $\begin{array}{l}\text { No of } \\
\text { infants }\end{array}$ \\
\hline None & 5 \\
Metaplastic epithelium only & 18 \\
Metaplastic epithelium + squamous debris & 16 \\
Metaplastic epithelium + 'squamous polyps' & 11 \\
Metaplastic epithelium + Drusen-like nodules & 4 \\
Metaplastic epithelium + inflammatory exudate & 10 \\
Metaplastic epithelium + damage to incus, & 8 \\
stapes, and incudo-stapedial joint & \\
\hline
\end{tabular}

survey (67 of 72), the epithelium had become converted into a complex structure with columnar ciliated epithelium, goblet cells, mucous cysts, and even mucous glands. The altered mucoperiosteum resembled the mucosa of the eustachian tube. In 18 of the 67 infants this glandular metaplasia was the sole abnormality, but glandular metaplasia was present in all 67 infants accompanying the additional features present.

Persistent aggregates of anucleate squames could be identified in a total of 27 infants, and had evoked a giant cell response. In one infant, step sectioning through the block showed incorporation of the squamous debris into a discrete squamous nodule buried deep within the mucoperiosteum. The pattern and outcome of the reaction to the squames depended on the amount present. Small amounts appear to be ingested by giant cells and soon removed, leaving residual foci of giant cells and metaplastic epithelium. This pattern was seen in 16 of the 27 infants. Larger volumes of squamous debris were encapsulated by macrophages and giant cells, and, in turn, became attached to either the tympanic membrane or the occicles, particularly the head of stapes. These aggregates formed polypoidal nodules resembling amnion nodosum and were identified in 11 of the 27 infants. Three of these infants have been reported previously. $^{3}$

In 4 infants, in addition to the presence of areas of glandular metaplastic epithelium, small drusen-like nodules ${ }^{\mathbf{1}} \mathbf{3}$ were identified. These lesions in their fully developed form consisted of small, hyaline, acellular nodules of tissue. They appeared to be derived from

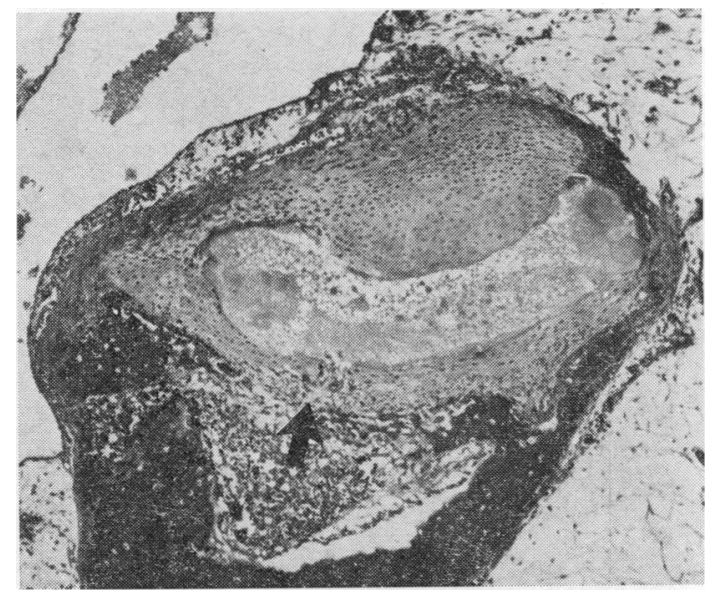

Fig. 1 An effusion (arrow) within the incudo-stapedial joint is shown. Note the area of subchondral bone resorption in the stapes (arrow). 36 week, 146 day infant. Attwood's, $\times 70$. 
organisation of amorphous proteinaceous material into the mucoperiosteum, thereby trapping small aggregates of nuclear debris.

In 10 infants otitis media with a purulent exudate

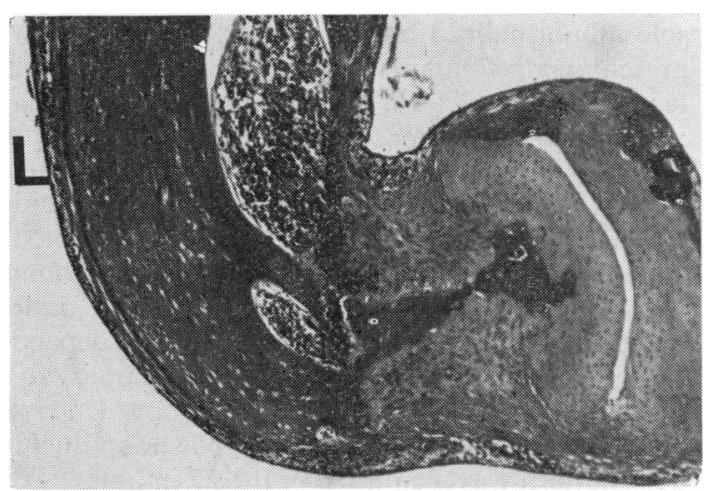

Fig. 2 (a) The normal configuration of the incudo-stapedial joint is shown. The long process of the incus $(L)$ takes a sharp right-angle bend into the lentiform process, which articulates with the stapes (S). 39 week, 17 day infant. Attwoot's, $\times 70$.

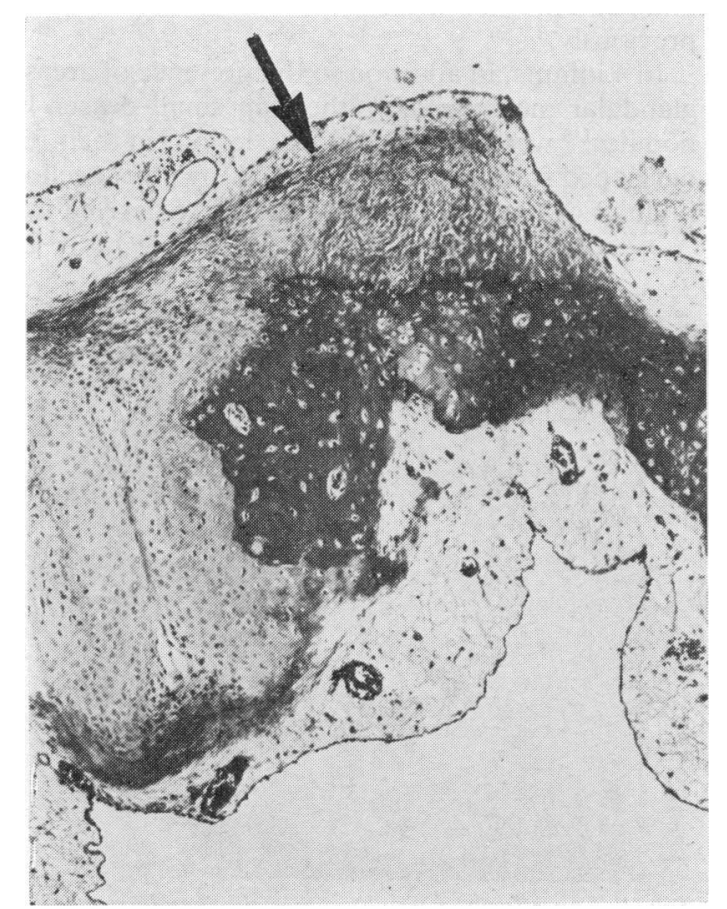

Fig. 2 (b) A subcapital fracture of the stapes with callus formation (arrow). 28 week, 130 day infant. Attwood's, $\times 80$. was present. The exudates filled the middle ear, and were associated with an interstitial reaction within the mucoperiosteum on both medial walls and over the ossicles. Glandular transformation of the mucoperiosteum was a constant accompaniment in all 10 infants. As in a previous study, ${ }^{1}$ otitis media was always associated with pneumonia. Of the 10 infants with otitis media, the tympanic membrane had been examined in 3 cases and was stated to be intact.

Destructive lesions. In 8 infants evidence of damage and destruction to the ossicles was noted. In one infant an effusion was present in the incudo-stapedial joint associated with subchondral bone resorption of the head of the stapes (Fig. 1). In 2 other infants extensive subchondral resorption of the stapedial head was the sole abnormality. In 3 infants destruction of the lentiform process of the incus was associated with fracture dislocation. In one of these infants a second fracture, presumably through an area of subchondral bone resorption, was identified in the head of the stapes. This second fracture was associated with early callus formation (Fig. 2).

In 2 infants the stapes had been destroyed. In the first infant, abundant granulation tissue filled the intercrural space and this was accompanied by subchondral bone resorption of the head and destruction of the anterior crus (Fig. 3). The granulation tissue contained abundant foamy macrophages, and a surprising finding was the presence of a small collection of anucleate squames embedded among them.

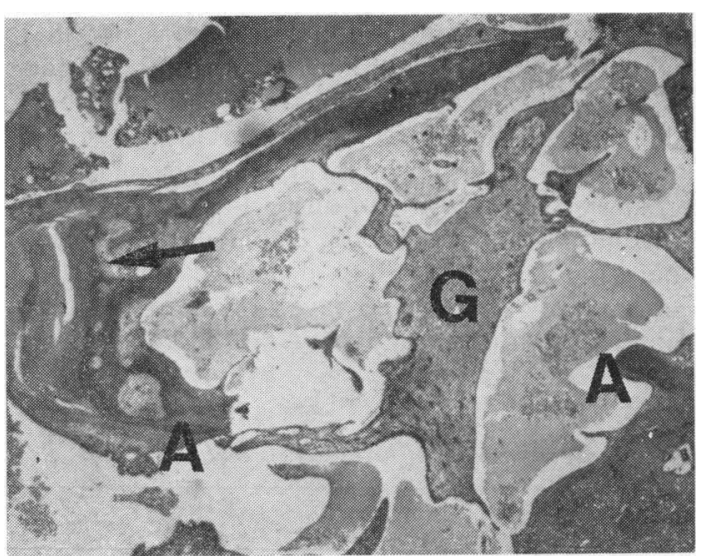

Fig. 3 (a) Extensive destruction of the stapes is seen. Granulation tissue $(G)$ fills the intercrural space. Subchondral resorption of bone is seen in the head (arrow) and the anterior crus is disrupted (ends of crus shown as A). 29 week, 243 day infant, Attwood's, $\times 35$. 


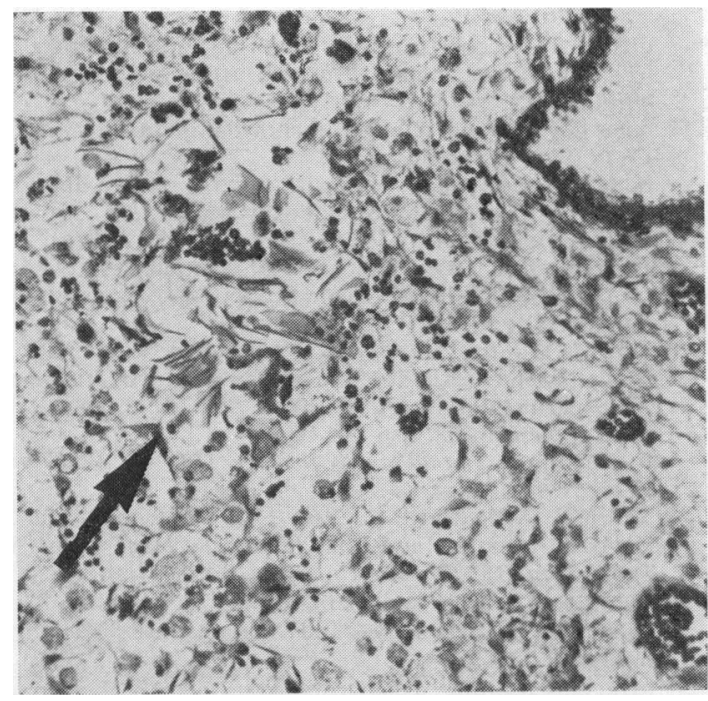

Fig. 3 (b) Anucleate squames (arron) embedded among foamy macrophages in the granulation tissue shown in Fig. 3a. Attwood's, $\times 120$.

In the last and oldest infant in this group, the method of examination of the right petrous temporal bone had been modified. The tegmen tympani was removed and abundant friable debris was seen. This

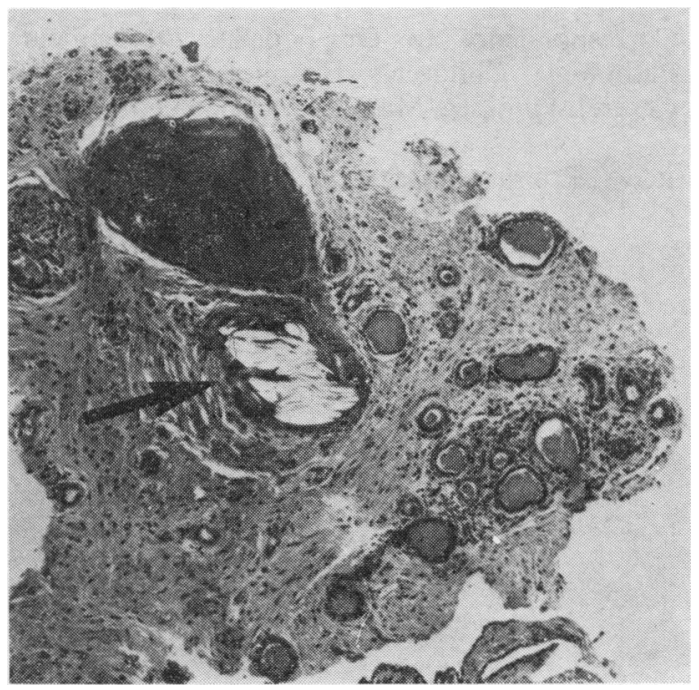

Fig. 4 No incudostapedial joint was seen on opening tegmen tympani. Structures identified in the vicinity of the incudo-stapedial articulation include a fragment of bone, cholesterol granuloma (arrow), and metaplastic ands, embedded in fibrous tissue. 33 weeks;

6 day infant. Attwood's, $\times 45$. was removed, and representative samples were studied using smears or sections. The smears showed the presence of foamy macrophages, similar to those identified in the other case with destruction of the stapes. Even after removal of the debris the stapes could not be delineated satisfactorily. In its site small fragments of bone embedded in fibrous tissue with cholesterol granulomata and metaplastic glands were identified (Fig. 4). These were interpreted as the remnants of the stapes.

\section{Discussion}

It is clear that a wide range of histological abnormalities can be identified within the middle ears of neonatal infants. They vary from metaplastic epithelial lesions, that may not produce any significant damage per se, to very florid inflammatory lesions and even destruction of ossicles. The metaplastic epithelial changes, in particular the glandular transformation, resemble those produced by obstruction of the eustachian tube in experimental animals. ${ }^{5}$ It is believed $^{6}$ that the development of mucous glands within the mucoperiosteum represents the basic lesion of secretory otitis media, and it is of interest that it was such a common finding in this series. Presumably, had these infants survived, they may have been predisposed to otitis media.

It is possible that the polypoidal, amnion nodosum-like lesions could interfere with movement of the ossicles. Certainly the large accumulations of squamous debris within the middle ear could predispose to the subsequent development of cholesterol granulomata as suggested by the infant illustrated in Fig. 4. Granulomatous reactions to squamous debris are well known in other sites such as ruptured epidermal cysts of skin. The persistence of squamous debris in the middle ear of the newborn may be relevant to the later development of cholesteatoma and epidermoid cysts at this site.

Frank otitis media, associated with an intact tympanic membrane, was a surprising finding especially since it was not diagnosed clinically. The symptoms and signs may have been masked by the concomitant antibiotic therapy many of these infants were receiving for their clinically detected pneumonia, but it has been shown that otitis media in the newborn may be present with an intact tympanum ${ }^{7}$ and that alterations in the light reflex are important as an early sign. ${ }^{8}$ The atypical presentation of otitis media in the newborn needs to be stressed.

Owing to interference with their blood supply, otitis media could have contributed to the destruction of the ossicles. The blood supply to the lentiform process of the incus and the head of the stapes is 
known to be extremely finely balanced and therefore vulnerable to a variety of insults. ${ }^{910}$ Certainly an inflamed mucoperiosteum could jeopardise the blood supply of the ossicles.

Several factors may affect the development of the lesions seen in our infants. Covert infections, aspirated amniotic squamous debris, ${ }^{1-3}$ the effects of oxygen, and obstruction of the eustachian tube by a nasal airway could be implicated to a variable extent in any one infant. The relative contribution of each is uncertain, and cannot be assessed in a retrospective necropsy study. It is obvious however, that a wide range of pathological findings may be identified in the middle ears of neonatal infants, particularly those who have required prolonged intensive care in the neonatal period. It is conceivable that similar changes, albeit less severe, may be present in survivors. Such infants might be expected to be at risk from the development of secretory otitis media or may even present with some degree of conductive hearing loss. Recently there has been increasing interest in the effect of conductive hearing loss on subsequent sensorineural hearing deficit in adults. ${ }^{11} 12$ Similar problems could be encountered in infants.

Neonatologists have not always directed much attention to the middle ear. Pathologists are not blameless either, since histological examination of the petrous temporal bone is not a routine procedure in many hospitals. Obviously, this situation needs to be remedied.

I thank Marion Leskovec, Sharryn Putns, and Stephanie Whittaker for technical assistance; Dr J Watts and Dr J Sinclair for encouragement; Dr D Hitch for help with the dissection of the petrous temporal bone in the last case.
References

1 deSa D J. Infection and amniotic aspiration of middle ea in stillbirths and neonatal deaths. Arch Dis Child 1973CP 48: 872-80.

2 deSa D J. Adrenal changes in chorioamnionitis. Arch $D$ 융 Child 1974; 49: 149-51.

3 deSa D J. Polypoidal organization of aspirated amnioti squamous debris (amnion nodosum) in middle ear cavit\$ of newborn infants. Arch Dis Child 1977; 52: 148-51.

4 Putns S, deSa D J. Application of a modified Attwood stain to the study of decalcified bone sections. J Cliz Pathol 1977; 30: 900-3.

5 Kowata I, Shibahara Y, Kaku Y, Wada J, Kamawoto K户 Epithelial changes in or of the middle ear mucoperiosteunf An experimental study. Ann Otol Rhinol Laryngol 1980. 89: Supplement 68, 236-40.

6 Friedmann I. Pathology of the ear. Oxford: Blackwelf 1974: 76-83.

7 Shurin P A, Pelton S I, Klein J O. Otitis media in the newborn infant. Ann Otol Rhinol Laryngol 1976; 850 Supplement 25, 216-22.

8 Bland R D. Otitis media in the first six weeks of life diagnosis, bacteriology, and management. Pediatrics 1972; 49: 187-97.

9 Brownlie-Smith A. An anatomical problem of the surgica treatment of otosclerosis. Proc R Soc Med 1966; 59 229-35.

10 Alberti P W R M. The blood supply of the long process of the incus and the head and neck of the stapes. $J$ Laryngeb Otol 1965; 79: 964-70.

11 Paparella M M, Brady D R. Sensorineural hearing loss iף chronic otitis media and mastoiditis. Trans Am Acas Ophthalmol Otolaryngol 1970; 74: 108-15.

12 English G M, Northern J L, Fria T J. Chronic otitis medi as a cause of sensorineural hearing loss. Arch Otolaryng $1973 ; 98: 18-22$.

Correspondence to $\mathrm{Dr} \mathrm{J}$ deSa, Department ox Pathology, Children's Hospital, Health Sciences Centre, Winnipeg, Manitoba, Canada R3E OW1.

Received 20 August 1982 